\title{
Simulation of UHE muons propagation for GEANT3
}

\author{
S. Bottai * \\ Dipartimento di Fisica, Università di Firenze and \\ INFN Sezione di Firenze \\ L. Perrone \\ Dipartimento di Fisica, Università di Lecce and \\ INFN Sezione di Lecce
}

\begin{abstract}
A simulation package for the transport of high energy muons has been developed. It has been conceived to replace the muon propagation software modules implemented in the detector simulation program GEANT3. Here we discuss the results achieved with our package and we check the agreement with numerical calculations up to $10^{8} \mathrm{GeV}$.

Keywords: Muon transport, Monte Carlo simulation.

PACS numbers: 25.30.Mr, 02.50.Ng
\end{abstract}

\section{Introduction}

Underground and underwater detectors for neutrino astronomy require simulation tools capable to correctly handle the propagation of high energy muons up to $\mathrm{PeV}$ energy region and above. The new object-oriented $(\mathrm{C}++)$ version of the detector simulation tool GEANT (GEANT4 [1]), will probably

*Address for corrispondence: Dipartimento di Fisica di Firenze and INFN Sezione di Firenze, Largo E.Fermi n. 2, I-50125 Firenze (Italy) tel. +39-055-229420, fax +39-055229330 
be suitable for this goal.

However many experiments [2, 3, 4, 5] still make use of GEANT3 [6] to simulate the detector response. Even if such package can in principle perform the simulation of particle propagation above $10 \mathrm{TeV}$, it has been mainly designed for accelerator experiments, whose tipical energy range doesn't exceed the $T e V$ region.

The simulation of muonic interactions is actually guaranteed by the authors for muon energy below $10 \mathrm{TeV}$ [6]. This is due to the parametrizations of cross sections for radiative processes contained in GEANT3, which are reliable only for energy up to $10 \mathrm{TeV}$. Moreover the description of photonuclear interaction is realized in a frame which significantly disagrees with theoretical calculations, for each muon energy.

Our new simulation code (GMU) has been carried out to make the GEANT3 standard library reliable in reproducing UHE muons propagation through matter.

GMU replaces the simulation of the radiative muonic interactions performed by GEANT3, for each energy and for each material, keeping the same structure of the original program. The procedure to apply the new code is completely transparent in such a way that no adjustments must be implemented in the programs that make use of the standard GEANT3 library.

\section{Radiative Cross sections in GEANT3 and GMU}

High-energy muons propagating through matter mainly interact by quasicontinuous (ionization) and discrete (bremsstrahlung, direct electron-positron pair production, photonuclear interaction) energy losses mechanisms. Ionization dominates at energy lower than few hundreds of $G e V$ while above the $\mathrm{TeV}$ region energy losses are mainly due to radiative processes.

The radiative processes are simulated stocastically by GEANT3 above a fixed (user supplied) transferred energy threshold; below this threshold they are treated as continuous. The models which describe the interaction processes are the physical input for the code. In more detail, simulating a given process requires:

- To evaluate the probability of occurrence of the process by sampling 
the total cross section of the process

- To generate the final state after interaction by sampling the differential cross section of the process

The reliability of the simulation is then affected both by the formulas (and/or parametrizations) chosen for cross sections and by the alghoritms used for sampling and for numerical integration.

Standard reference formulas for UHE calculations can be found in the paper by Lohmann and Voss 10 which also tabulate the average muon energy losses for many materials and compounds up to $10 \mathrm{TeV}$.

Direct electron pair production differential cross section has been first calculated by Kelner and Kotov in the framework of QED theory [7]. We have used the well-known parametrization performed by Kokoulin and Petrukhin [8] which considers the corrections for atomic and nuclear form factors.

For bremsstrahlung differential cross section we have used the formula derived by Andreev and Bugaev [9] which takes into account the structure of nuclear target (elastic and inelastic form factors) and the exact contributions due to atomic electrons (screening effect and bremsstrahlung of the muons on electrons). The formula used by Lohmann and Voss had been carried out just from this one by Petrukhin and Shestakov [1] by neglecting bremsstrahlung on electrons and nuclear effects in light materials $(Z<10)$. The discrepancy between the two approaches reaches few percents, in terms of bremsstrahlung energy losses, in the worse case (for materials with $Z \sim 10$ ) and it is almost always negligible in the calculation of the total muon energy losses.

For the photonuclear interaction we have used the differential cross section calculated by Bezrukov and Bugaev [12] within the vector meson dominance hypothesis. We also have considered the recent accelerator data coming from ZEUS and H1 [13] [14] according to the ref. [15]. For this process we have not considered, at present, the angle between the ingoing and outgoing muon, taking the reasonable approximation of completely forward scattering. As a consequence, the code is not suitable for studies dedicated to muon-nucleus scattering at large angles.

Total cross section and average energy loss for each radiative process $k$ can be calculated, starting from differential cross sections, as follows: 


$$
\begin{gathered}
\sigma_{\text {Tot }}^{k}=\int_{v m i n}^{v m a x} \frac{d \sigma^{k}}{d v}(v, E) d v \\
-\left\langle\frac{d E}{d x}\right\rangle_{k}=\frac{N_{A}}{A} E \int_{v \min }^{v m a x} v \frac{d \sigma^{k}}{d v}(v, E) d v
\end{gathered}
$$

- $N_{A}$ and $A$ are Avogadro's number and the mass number respectively

- $v$ is the fraction of initial energy $E$ lost by muon at the occurrence of the process $k$

- vmin and vmax are the kinematical limits for the allowed values of $v$ (匹12, ,10])

- $x$ is the thickness of throughgone matter, expressed in $\mathrm{g} / \mathrm{cm}^{2}$

$$
\frac{d \sigma^{k}}{d v}(v, E)
$$

is the differential cross section for the process $k$ (explicit formulas are given in the Appendix)

The calculation of energy losses performed with the formulas described above agree with the Lohmann and Voss results at percent level in the energy range they considered $(1 \mathrm{GeV}-10 \mathrm{TeV}$.)

In fig. 11 we report the total cross sections for the radiative processes as they are tabulated at the initialization procedures by standard GEANT321 and by GEANT321 plus GMU.

In the first case the points are calculated with approximated analytical parametrizations whose accuracy is guaranteed (at least for bremsstrahlung and pair production) within $5 \%$ in the declared energy range $(E<10 \mathrm{TeV})$. In the second case the points are calculated by performing numerical integration of the formulas given in the Appendix. (This operation requires a small amount of CPU time at the initialization, depending also on the number of materials considered.) 
The photonuclear cross sections differ by more than one order of magnitude. This discrepancy has already been observed in previous works 16 and it is expected to affect the total energy loss especially in the case of light materials. The total cross sections for bremsstrahlung and pair production below $100 \mathrm{TeV}$ seem to be in excellent agreement but for energy above 100 $\mathrm{TeV}$ the GEANT321 cross sections strongly deviate from expectation.

\section{$3 \quad$ Simulation of muon energy loss}

In order to evaluate the correctness of the simulation code we have performed some checks for different materials. For each test run we have activated the full stochastical regime and we have evaluated the average energy loss resulting from the simulation. The values achieved with standard GEANT321 and with GEANT321 plus GMU have been compared with numerical calculations of the same quantities [see eq.(2)].

In fig. 2 and 3 we present the results for water and for standard rock respectively. The simulation performed by GEANT321 plus GMU reproduces very well the numerical calculation at any energy, both for total energy losses and for the individual processes.

For such materials, the GEANT321 simulation is in good agreement up to $100 \mathrm{TeV}$, but it produces much greater average energy loss for energy exceeding $100 \mathrm{TeV}$.

In table 1 we report the total and the photonuclear energy losses in hydrogen as they result from simulation performed by the two codes $(\triangle \Longrightarrow$ GEANT321; $ઋ$ GEANT321+GMU). The discrepancy is not negligible, even at low energy, being around $20 \%$ at $10 \mathrm{TeV}$. The main source of this disagreement is clearly due to a different estimation of the photonuclear process, whose relative importance grows for light materials.

In order to further verify the reliability of the simulation code we performed some specific runs by recording the fraction $v$ of energy lost by muon at the occurrence of each process. (Indeed the average energy loss is not especially sensitive to very big or very small energy transfer).

The distributions of such variable have been compared with analytical expressions for differential cross sections. The results are shown in fig. 4 , 5 
and 6 for a fixed muon energy $(E=100 \mathrm{TeV})$ : they confirm the correctness of the sampling performed by GMU.

\section{Conclusions}

The simulation of muon propagation carried out by standard GEANT3 seems to be out of control for energy above $10^{5} \mathrm{GeV}$.

Moreover GEANT3 slightly underestimates the energy losses for very light materials (at lower energies too), due to a strong discrepancy between expected and simulated values of the muon photonuclear cross section.

GEANT321 in addition to the new code GMU performs complete agreement with expectation in the tested energy range (up to $10^{8} \mathrm{GeV}$ ).

Further improvements will consist in a more accurate simulation of the direction of outgoing particle produced in photonuclear interaction and in taking into account the influence of the medium (LPM effect) wich becomes important in the process of muon bremsstrahlung at extreme high energy. In this region direct muon pair production by muons should be taken into account too.

The GMU code can be requested by conctacting the authors. E-mail:

- Bottai@fi.infn.it

- Lorenzo@le.infn.it

\section{Acknowledgments}

We would like to thank the members of the Lecce MACRO group and prof. V.A.Naumov for useful discussions and concrete help.

\section{References}

[1] The Geant4 Object Oriented Simulation Toolkit M.G. Pia for the Geant4 Collaboration, EPS-HEP99 Conference,Tampere, 1999 (see also http://wwwinfo.cern.ch/asd/geant4); 
[2] MACRO collaboration Nucl. Inst. and Meth. A 324337 (1993);

[3] S. Bottai for the NESTOR collaboration Indirect search for Dark Matter with NESTOR, Proceedings of IDM98 World Scientific pg. 508;

[4] L. Moscoso for the Antares collaboration Expected Performance of the ANTARES Experiment (ICRC 99), Salt Lake City, UT, 17-25 Aug 1999;

[5] Wiebush C. 1995 The detection of faint light in deep underwater neutrino telescopes Ph. D. thesis;

[6] GEANT - Detector Description and Simulation Tool CERN Program Library Long Writeup W5013;

[7] S.R. Kelner and V.V. Kotov, Sov. J. Nucl. Phys. 7237 (1968);

[8] R.P. Kokoulin and A.A. Petrukhin Proceedings of the 12th International Cosmic Ray Conference, Hobart, 1971 Vol 6, pg. A 2436;

[9] Yu. M. Andreev and E.V. Bugaev, Phys. Rev. D 55, 1233 (1997);

[10] W. Lohmann, R. Kopp and R. Voss CERN yellow report 85-03 (1985);

[11] A.A. Petrukhin and V.V. Shestakov, Can. J. Phys. 46 S377 (1968);

[12] L.B. Bezrukov and E.V. Bugaev, Sov. J. Nucl. Phys. 33635 (1981);

[13] M. Derrick et al. (ZEUS Collaboration) Phys. Lett. B 293465 (1992);

[14] T. Ahmed et al. (H1 Collaboration) Phys. Lett. B 299374 (1993);

[15] E. V. Bugaev, A. Misaki, V. A. Naumov, T. S. Sinegovskaya, S. I. Sinegovsky, and N. Takahashi Phys. Rev. D 58, 054001 (1998);

[16] Battistoni, Forti, Ferrari, Scapparone INFN/AE-97/10 1997;

[17] A. Donnachie and P.V. Landshoff Phys. Lett. B 296227 (1992) 


\section{APPENDIX}

\section{Radiative cross sections formulae}

- $N_{A}$ is Avogadro's number

- $Z$ and $A$ are the atomic number and the atomic weight of the material

- $v$ is the fraction of initial energy $E$ lost in the interaction $\left(E^{\prime}=E(1-v)\right)$

- $\alpha$ is the fine structure constant

- $r_{e}$ is the classical electron radius

- $\lambda_{e}=r_{e} \alpha^{-1}$ is the electron Compton wavelength

- $m_{e}, m_{\mu}, m_{\pi}$ and $M_{p}$ are the electron, muon, pion and proton rest masses $(c=1)$ respectively

The formulae for radiative differential cross sections are taken from ref. [8] for $e^{+} e^{-}$pair-production, from ref. [9] for bremsstrahlung and from ref. [12] and [15] for photonuclear interaction.

\section{- PAIR PRODUCTION}

$$
\begin{gathered}
\frac{d \sigma_{p}}{d v d \rho}=\alpha^{4} \frac{2}{3 \pi}\left(Z \lambda_{e}\right)^{2} \frac{1-v}{v}\left[\Phi_{e}+\frac{m_{e}^{2}}{m_{\mu}^{2}} \Phi_{\mu}\right] \\
\Phi_{e}=\left\{\left[\left(2+\rho^{2}\right)(1+\beta)+\xi\left(3+\rho^{2}\right)\right] \ln \left(1+\frac{1}{\xi}\right)\right. \\
\left.+\frac{1-\rho^{2}-\beta}{1+\xi}-\left(3+\rho^{2}\right)\right\} S_{e}
\end{gathered}
$$




$$
\begin{gathered}
\Phi_{\mu}=\left\{\left[\left(1+\rho^{2}\right)\left(1+\frac{3 \beta}{2}\right)-\frac{1}{\xi}(1+2 \beta)\left(1-\rho^{2}\right)\right] \ln (1+\xi)\right. \\
\left.+\frac{\xi\left(1-\rho^{2}-\beta\right)}{1+\xi}+(1+2 \beta)\left(1-\rho^{2}\right)\right\} S_{\mu} \\
S_{e}=\ln \frac{R Z^{-1 / 3} \sqrt{(1+\xi)\left(1+Y_{e}\right)}}{1+\frac{2 m_{e} \sqrt{e} R Z^{-1 / 3}(1+\xi)\left(1+Y_{e}\right)}{E v\left(1-\rho^{2}\right)}} \\
-\frac{1}{2} \ln \left[1+\left(\frac{3}{2} \frac{m_{e}}{m_{\mu}} Z^{1 / 3}\right)^{2}(1+\xi)\left(1+Y_{e}\right)\right] \\
S_{\mu}=\ln \frac{(2 / 3)\left(m_{\mu} / m_{e}\right) R Z^{-2 / 3}}{1+\frac{2 m_{e} \sqrt{e} R Z^{-1 / 3}(1+\xi)\left(1+Y_{\mu}\right)}{E v\left(1-\rho^{2}\right)}} \\
Y_{e}=\frac{5-\rho^{2}+4 \beta\left(1+\rho^{2}\right)}{2(1+3 \beta) \ln \left(3+\frac{1}{\xi}\right)-\rho^{2}-2 \beta\left(2-\rho^{2}\right)} \\
Y_{\mu}=\frac{4+\rho^{2}+3 \beta\left(1+\rho^{2}\right)}{\left(1+\rho^{2}\right)\left(\frac{3}{2}+2 \beta\right) \ln (3+\xi)+1-\frac{3}{2} \rho^{2}} \\
\xi=\frac{m_{\mu}^{2} v^{2}}{4 m_{e}^{2}} \frac{\left(1-\rho^{2}\right)}{(1-v)} ; \beta=\frac{E^{2}}{2(1-v)}
\end{gathered}
$$

The integration limits are:

$$
\begin{gathered}
\frac{4 m_{e}}{E} \leq v \leq 1-\frac{3 \sqrt{e}}{4 E} m_{\mu} Z^{1 / 3} \\
0 \leq|\rho| \leq\left(1-\frac{6 m_{\mu}^{2}}{E^{2}(1-v)}\right) \sqrt{1-\frac{4 m_{e}}{E v}}
\end{gathered}
$$

where: 
- $E^{+}$and $E^{-}$are the energies of the positron and electron

- $R=189$ is the radiation logarithm value

- $e=\exp (1)$

We have taken into account the influence of the atomic electrons replacing $Z^{2}$ by $Z(Z+1)$.

\section{- BREMSSTRAHLUNG}

$$
\begin{gathered}
\frac{d \sigma_{b}}{d v}=\alpha\left(\frac{1}{v}\right)\left(2 r_{e} Z \frac{m_{e}}{m_{\mu}}\right)^{2}\left\{\left(1+\frac{E^{\prime 2}}{E^{2}}\right) \Phi_{1}\left(q_{\min }, Z\right)-\frac{2}{3} \frac{E^{\prime}}{E} \Phi_{2}\left(q_{\min }, Z\right)\right\} \\
\Phi_{1,2}\left(q_{\min }, Z\right)=\Phi_{1,2}^{0}\left(q_{\min }, Z\right)
\end{gathered}
$$

$$
\begin{aligned}
\Phi_{1}^{0}\left(q_{\text {min }}, Z\right)= & \frac{1}{2}\left(1+\ln \frac{m_{\mu}^{2} a_{1}^{2}}{1+x_{1}^{2}}\right)-x_{1} \arctan \frac{1}{x_{1}} \\
& +\frac{1}{Z}\left[\frac{1}{2}\left(1+\ln \frac{m_{\mu}^{2} a_{2}^{2}}{1+x_{2}^{2}}\right)-x_{2} \arctan \frac{1}{x_{2}}\right]
\end{aligned}
$$

$$
\begin{gathered}
\Phi_{2}^{0}\left(q_{\text {min }}, Z\right)=\frac{1}{2}\left(\frac{2}{3}+\ln \frac{m_{\mu}^{2} a_{1}^{2}}{1+x_{1}^{2}}\right)+2 x_{1}^{2}\left(1-x_{1} \arctan \frac{1}{x_{1}}+\frac{3}{4} \ln \frac{x_{1}^{2}}{1+x_{1}^{2}}\right) \\
+\frac{1}{Z}\left[\frac{1}{2}\left(\frac{2}{3}+\ln \frac{m_{\mu}^{2} a_{2}^{2}}{1+x_{2}^{2}}\right)+2 x_{2}^{2}\left(1-x_{2} \arctan \frac{1}{x_{2}}+\frac{3}{4} \ln \frac{x_{2}^{2}}{1+x_{2}^{2}}\right)\right], \\
\Delta_{1}\left(q_{\text {min }}, Z\right)=\ln \frac{m_{\mu}}{q_{c}}+\frac{a}{2} \ln \frac{a+1}{a-1}
\end{gathered}
$$




$$
\begin{gathered}
\Delta_{2}\left(q_{\min }, Z\right)=\ln \frac{m_{\mu}}{q_{e}}+\frac{a}{4}\left(3-a^{2}\right) \ln \frac{a+1}{a-1}+\frac{2 m_{\mu}^{2}}{q_{c}^{2}} \\
q_{\min } \simeq \frac{m_{\mu}^{2} v}{2 E(1-v)}, \quad x_{i}=a_{i} q_{\min }, \\
a=\sqrt{1+\frac{4 m_{\mu}^{2}}{q_{c}^{2}}, \quad q_{c}=1.9 m_{\mu} Z^{-1 / 3}}, \\
a_{1}=\frac{184.15}{\sqrt{e} Z^{1 / 3} m_{e}}, \quad a_{2}=\frac{1194.0}{\sqrt{e} Z^{2 / 3} m_{e}},
\end{gathered}
$$

- where $e=\exp (1)$

The integration limits are:

$$
0 \leq v \leq 1-\frac{3 \sqrt{e}}{4 E} m_{\mu} Z^{1 / 3}
$$

\section{- PHOTONUCLEAR INTERACTION}

$$
\begin{aligned}
\frac{d \sigma_{n}}{d v} & =\frac{\alpha}{8 \pi} A \sigma_{\gamma p}(\nu) v\left\{H(v) \ln \left(1+\frac{m_{2}^{2}}{t}\right)-\frac{2 m_{\mu}^{2}}{t}\right. \\
& +G(z)\left[H(v) \ln \left(1+\frac{m_{1}^{2}}{t}\right)-H(v) \frac{2 m_{1}^{2}}{m_{1}^{2}+t}-\frac{2 m_{\mu}^{2}}{t}\right] \\
& \left.+\frac{2 \xi m_{\mu}^{2}}{t}\left[G(z) \frac{2 m_{1}^{2}}{m_{1}^{2}+t}+\frac{m_{2}^{2}}{t} \ln \left(1+\frac{t}{m_{2}^{2}}\right)\right]\right\}
\end{aligned}
$$


$\nu=v E$ is the energy of virtual photon

$$
\begin{gathered}
H(v)=1-\frac{2}{v}=\frac{2}{v^{2}}, \quad G(z)=\frac{9}{z}\left\{\frac{1}{2}+\frac{1}{z^{2}}\left[(1+z) e^{-z}-1\right]\right\}, \\
z=0.00282 A^{\frac{1}{3}} \sigma_{\gamma p}(\nu), \quad t=\frac{m_{\mu}^{2} v^{2}}{1-v}, \\
m_{1}^{2}=0.54 \mathrm{GeV}^{2}, \quad \mathrm{~m}_{2}^{2}=1.80 \mathrm{GeV}^{2}, \quad \xi=0.25 .
\end{gathered}
$$

The differential cross section is proportional to the total cross section $\sigma_{\gamma N}$, for absorption of a real photon of energy $\nu=s / 2 m_{N}=v E$ by a nucleon. In this calculation we have used the Regge-type parametrization for $\sigma_{\gamma N}$, according ref. [17] This model performs the best fit to accelerator data $([13],[14)$.

$$
\sigma_{\gamma N}=\left[67.7 s^{0.0808}+129 s^{-0.4525}\right] \mu b
$$

The integration limits are:

$$
\frac{1}{E}\left(m_{\pi}+\frac{m_{\pi}^{2}}{2 M_{p}}\right) \leq v \leq 1-\left(1+\frac{m_{\mu}^{2}}{M_{p}^{2}}\right) \frac{M_{p}}{2 E}
$$




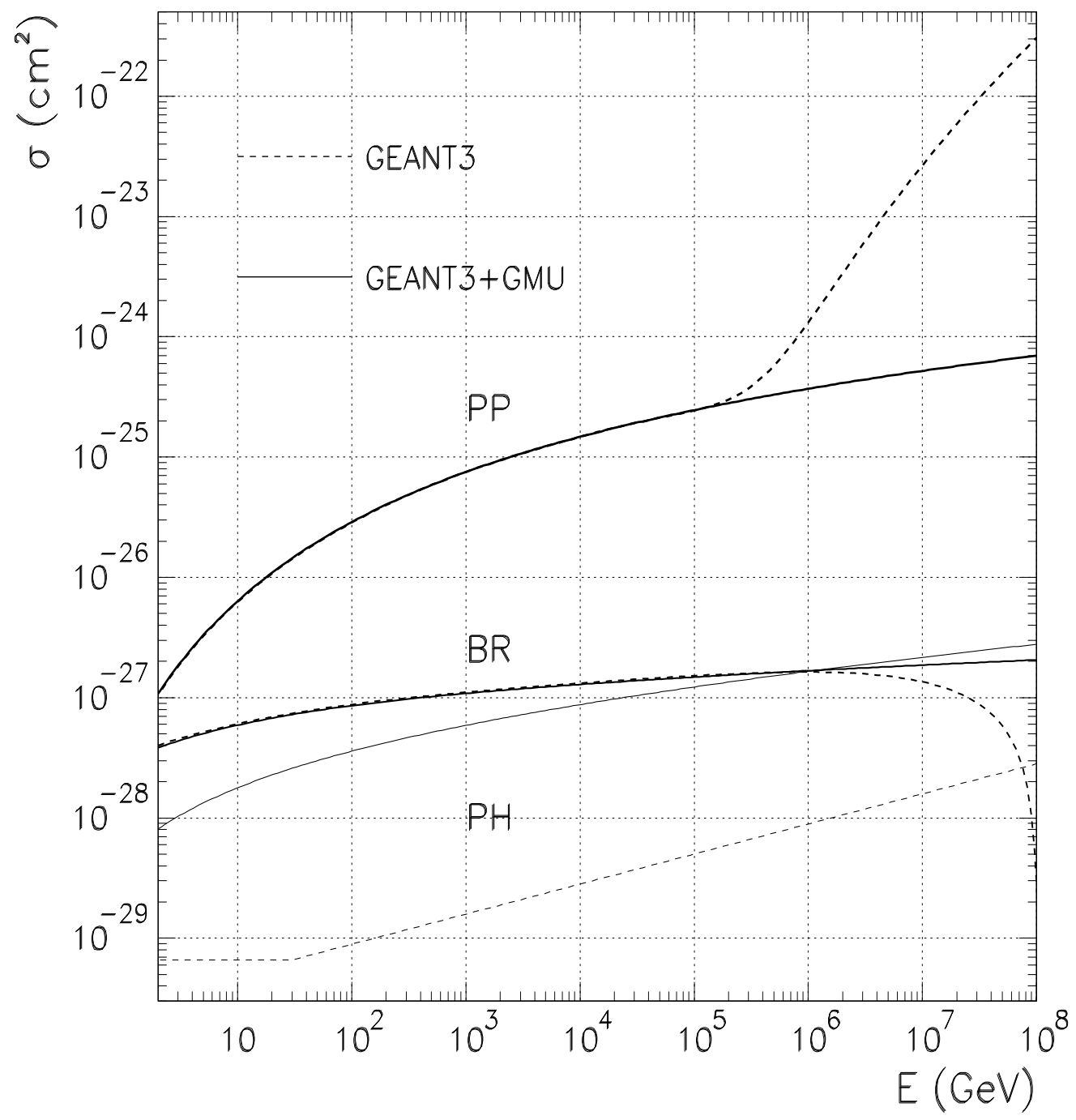

Figure 1: 


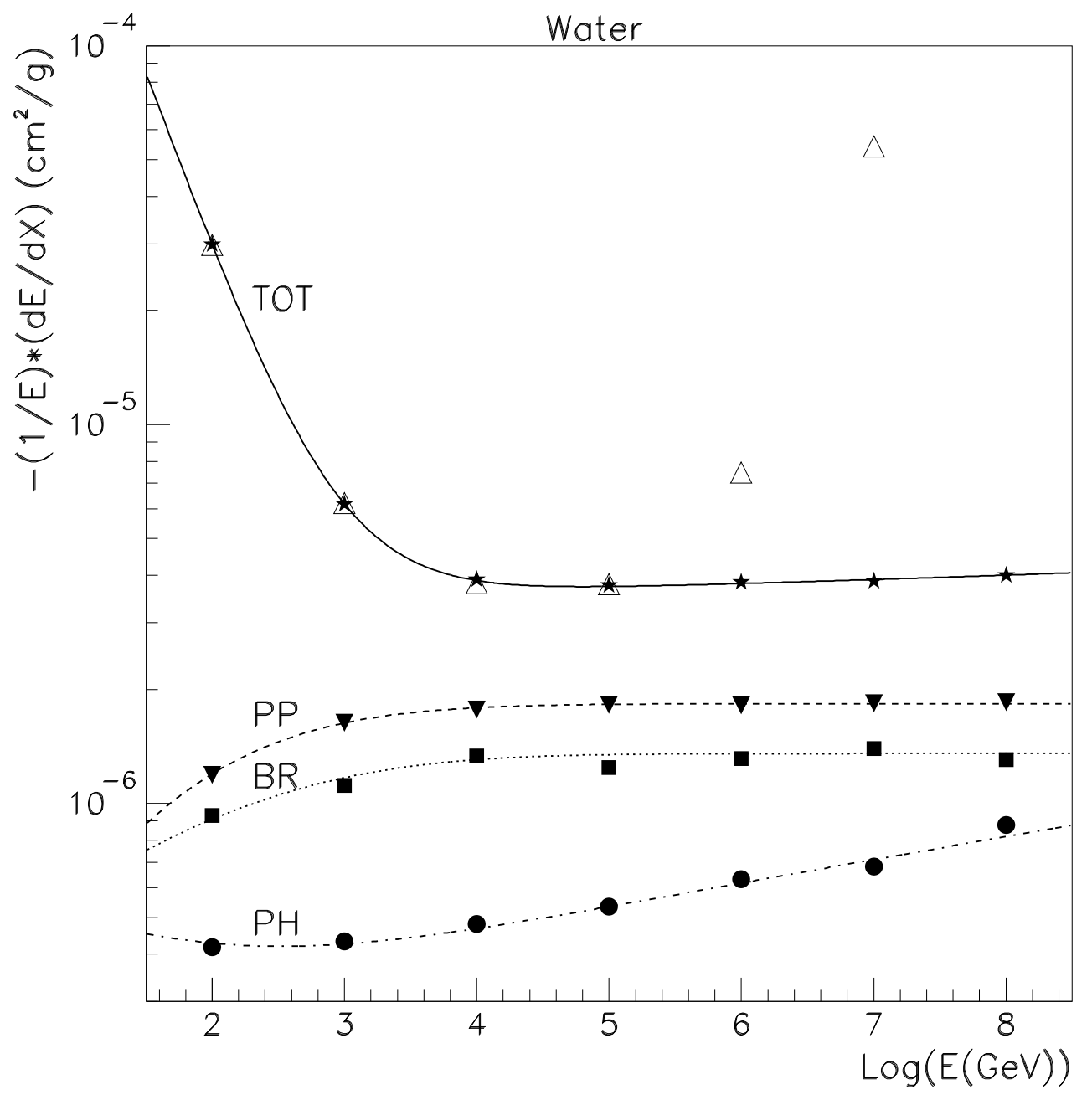

Figure 2: 


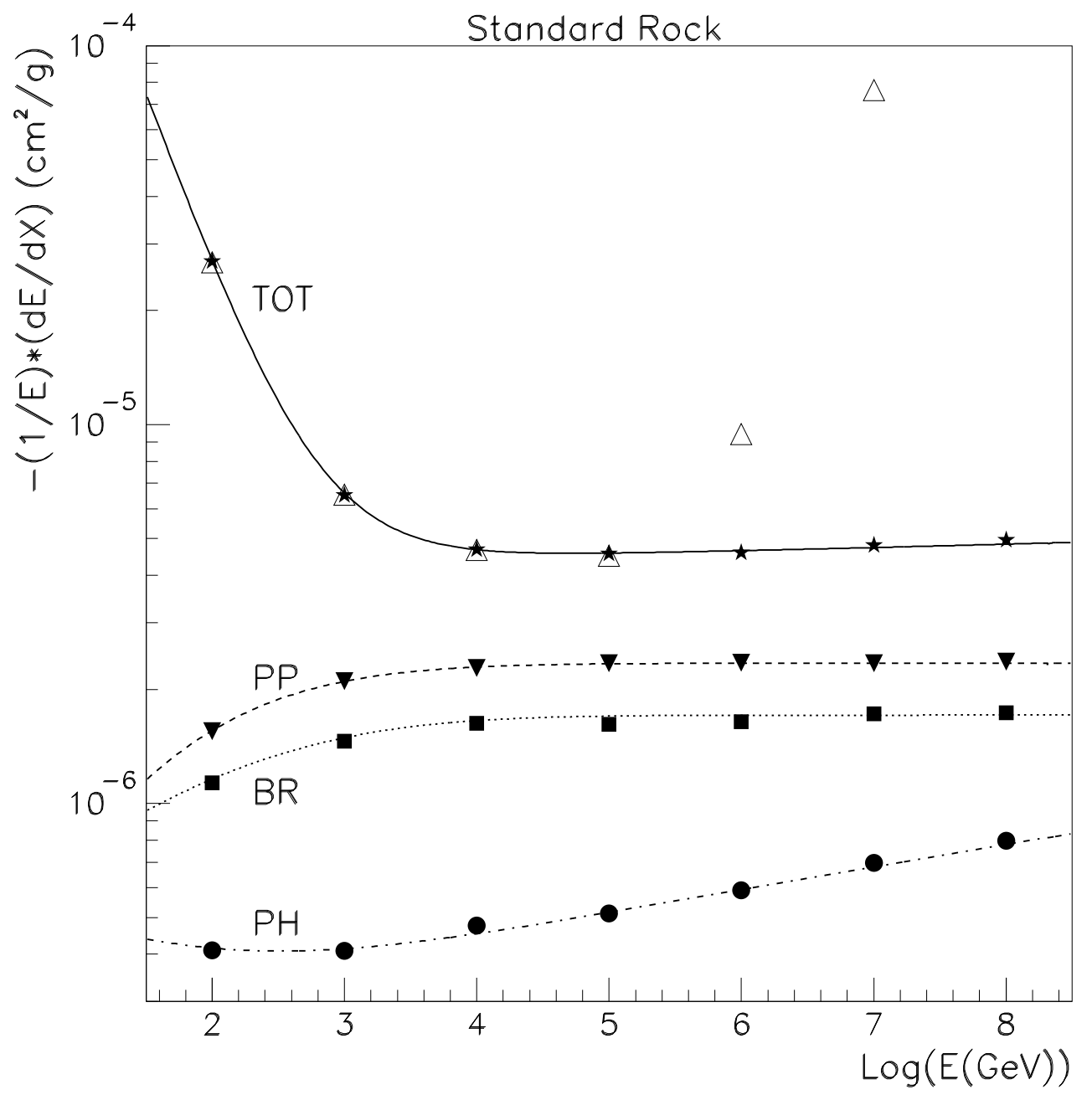

Figure 3: 


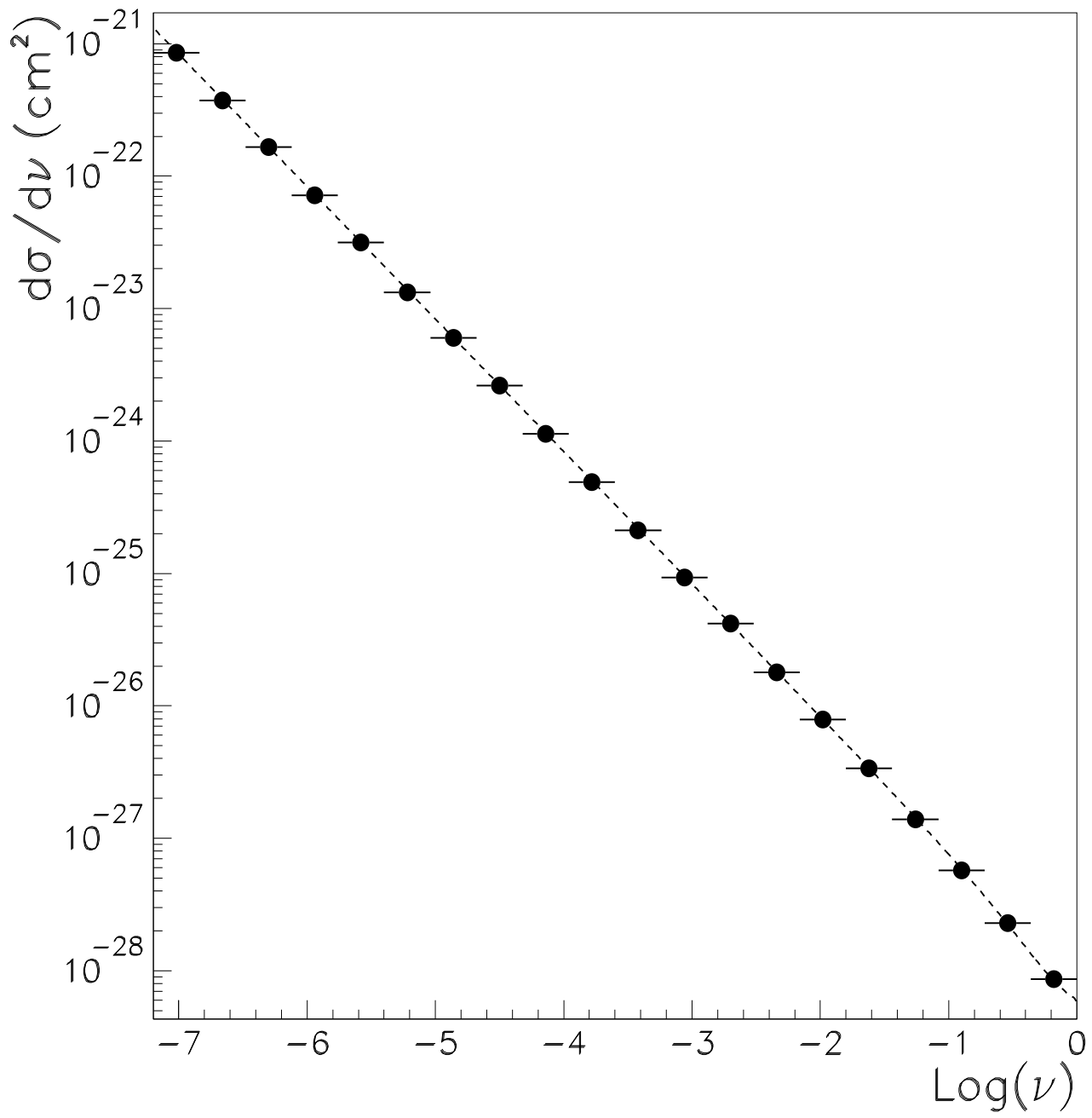

Figure 4: 


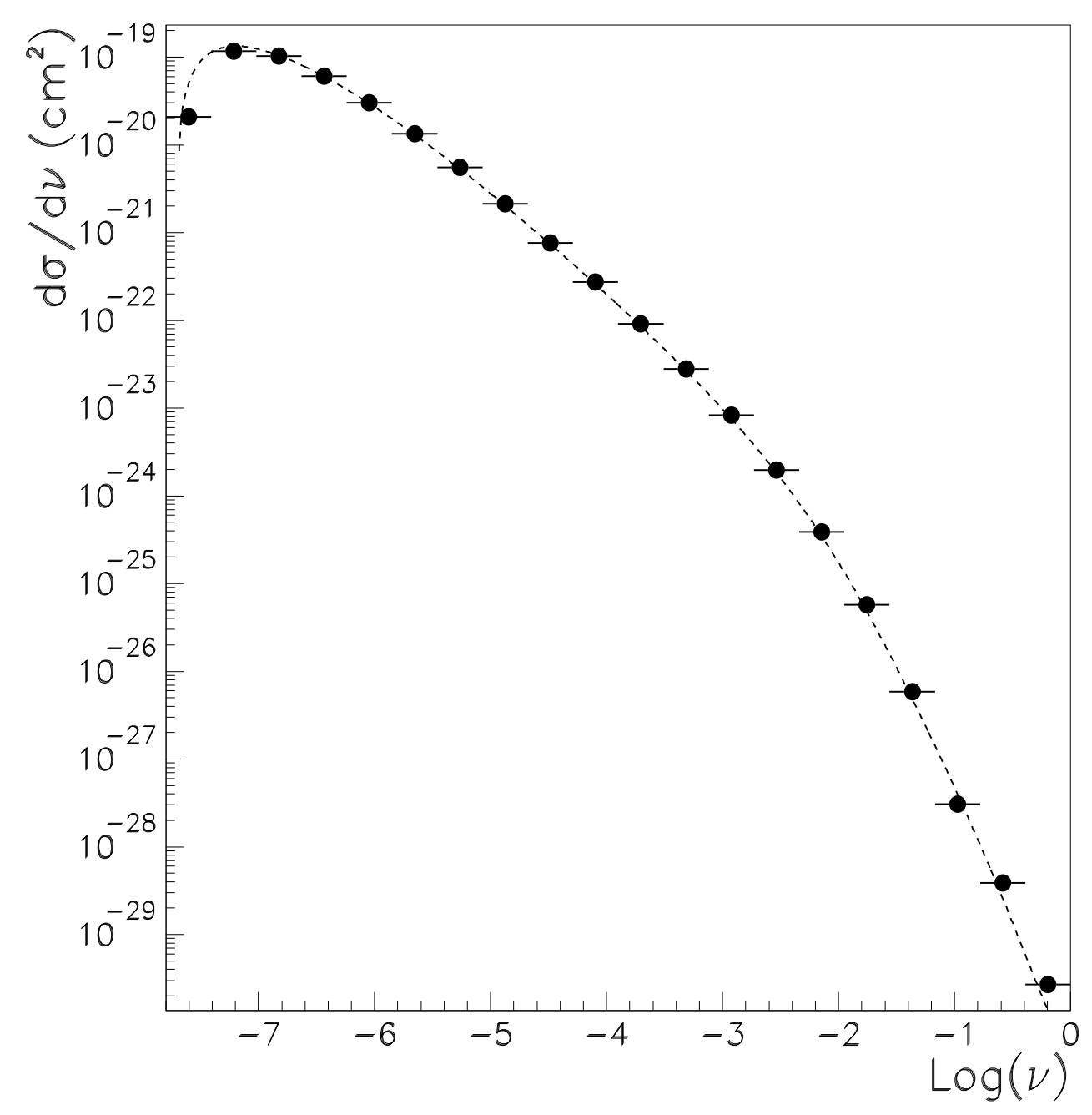

Figure 5: 


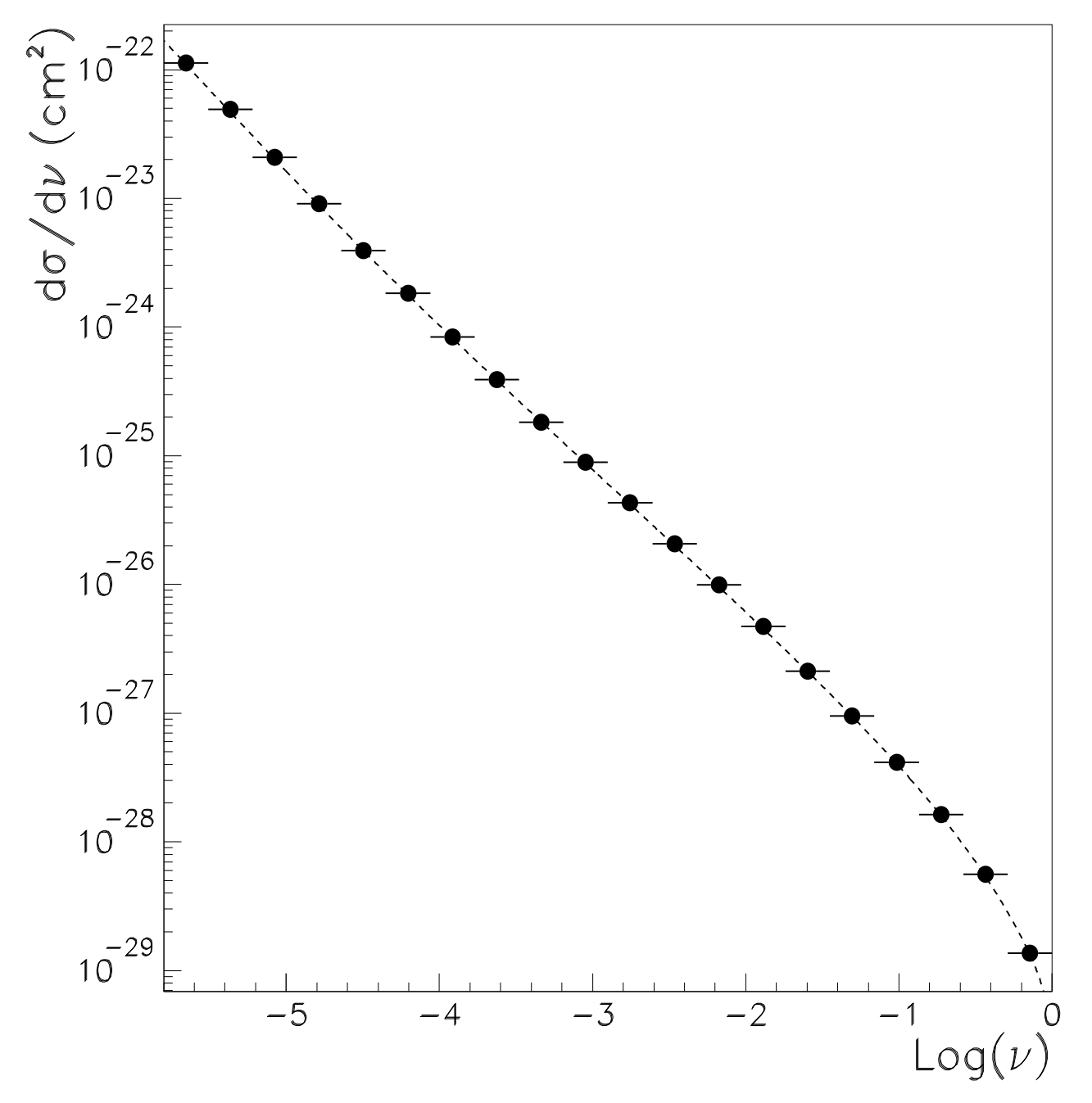

Figure 6: 
Figure 1: Total Cross Sections for radiative interaction processes vs muon energy $\left(\mathrm{PP} \rightarrow e^{+} e^{-}\right.$pair-production, $\mathrm{BS} \rightarrow$ bremsstrahlung, $\mathrm{PH} \rightarrow$ photonuclear interaction). The plot shows the values tabulated by GEANT321 (dashed lines) and by GEANT321 plus GMU (full lines). The material is Standard Rock $(Z=11, A=22)$.

Figure 2: Average total muon energy loss in Water $(Z / A=0.555)$ vs the $\log _{10} E$ ( $E$ is the muon energy). The plot shows the results from GEANT321 (empty triangles) and from GEANT321 plus GMU (full stars); the contributions from individual radiative processes (performed by GEANT321 plus GMU) and results from numerical calculations (lines) are also shown. Statistical error bars are comparable with dots size in case of photonuclear interactions $(\mathrm{PH})$ and bremsstrahlung $(\mathrm{BR})$.

Figure 3: Average total muon energy loss in Standard Rock $(Z=11$, $A=22$ ) vs the $\log _{10} E$ ( $E$ is the muon energy). The plot shows the results from GEANT321 (empty triangles) and from GEANT321 plus GMU (full stars); the contributions from individual radiative processes (performed by GEANT321 plus GMU) and results from numerical calculations (lines) are also shown. Statistical error bars are comparable with dots size in case of photonuclear interactions $(\mathrm{PH})$ and bremsstrahlung (BR).

Figure 4: Differential cross section for muon bremsstrahlung vs $\log _{10} v$ ( $v$ is the fraction of energy $E$ lost by muon in the interaction). The numerical calculation (dashed line) is compared with the result obtained from the simulation performed with the code GEANT321 plus GMU. The material is Standard Rock $(E=100 \mathrm{TeV})$.

Figure 5: Differential cross section for pair production vs $\log _{10} v$ ( $v$ is the fraction of energy $E$ lost by muon in the interaction). The numerical calculation (dashed line) is compared with the result obtained from the simulation performed with the code GEANT321 plus GMU. The material is Standard Rock $(E=100 \mathrm{TeV})$.

Figure 6:

Differential cross section for photonuclear interaction vs $\log _{10} v(v$ is the fraction of energy $E$ lost by muon in the interaction). The numerical calculation (dashed line) is compared with the result obtained from the simulation 
performed with the code GEANT321 plus GMU. The material is Standard Rock $(E=100 \mathrm{TeV})$. 


\begin{tabular}{||c|c|c|c||}
\hline \hline $\begin{array}{c}\mathrm{E} \\
(T e V)\end{array}$ & & $\begin{array}{c}(1 / E) \cdot(d E / d x)_{T o t} \\
\left(\mathrm{~cm}^{2} \cdot \mathrm{g}^{-1}\right)\end{array}$ & $\begin{array}{c}(1 / E) \cdot(d E / d x)_{\text {Phot }} \\
\left(\mathrm{cm}^{2} \cdot \mathrm{g}^{-1}\right)\end{array}$ \\
\hline \multirow{2}{*}{0.1} & $\triangle$ & $(5.270 \pm 0.005) \cdot 10^{-5}$ & $(0.0030 \pm 0.0001) \cdot 10^{-5}$ \\
\cline { 2 - 4 } & $\star$ & $(5.322 \pm 0.005) \cdot 10^{-5}$ & $(0.047 \pm 0.001) \cdot 10^{-5}$ \\
\hline \hline \multirow{2}{*}{1} & $\triangle$ & $(0.704 \pm 0.004) \cdot 10^{-5}$ & $(0.0062 \pm 0.0002) \cdot 10^{-5}$ \\
\cline { 2 - 4 } & $\star$ & $(0.738 \pm 0.004) \cdot 10^{-5}$ & $(0.046 \pm 0.001) \cdot 10^{-5}$ \\
\hline \hline \multirow{2}{*}{10} & $\triangle$ & $(0.224 \pm 0.002) \cdot 10^{-5}$ & $(0.0155 \pm 0.0005) \cdot 10^{-5}$ \\
\cline { 2 - 4 } & $\star$ & $(0.262 \pm 0.002) \cdot 10^{-5}$ & $(0.0515 \pm 0.001) \cdot 10^{-5}$ \\
\hline \hline
\end{tabular}

Table 1: Total energy losses for Hydrogen in comparison with the photonuclear interaction contributions. $\triangle \Longrightarrow$ GEANT321; $~ \Longrightarrow$ GEANT321 + GMU 